\title{
Inventário de mamíferos não voadores da reserva particular do patrimônio natural Mata da Serra (ES)
}

A Mata Atlântica se estende do Rio Grande do Norte até o Rio Grande do Sul na faixa litorânea. Ela pode ser dividida em dois corpos: Floresta Litorânea e Floresta de Montanha. Ambas possuem um grande número de endemismos e são detentoras de uma altíssima biodiversidade e de um valioso banco genético. O estabelecimento de Unidades de Conservação em áreas remanescentes deste bioma tem se tornado uma das principais estratégias para a manutenção da biodiversidade. Além da criação dessas unidades, também é necessário que sejam realizados estudos que estabeleçam a real situação de conservação ambiental das mesmas, colaborando para o aumento do conhecimento da biologia das espécies integrantes destas áreas. 0 objetivo deste estudo foi inventariar os mamíferos existentes na RPPN Mata da Serra, no município de Vargem Alta, Espírito Santo, como forma de subsidiar a criação de um Plano de Manejo da área e outras melhorias para a mesma. Em um período de 11 meses de campo (de setembro de 2012 a agosto de 2013), foram registrados 107 animais, divididos em 15 espécies de mamíferos não voadores e distribuídas em 05 ordens e 11 famílias. Das espécies registradas, três estão ameaçadas de extinção. Os resultados obtidos ressaltam a importância das unidades de conservação, mesmo aquelas de área relativamente pequena, para conectividade a manutenção e preservação da biodiversidade. Indicam que a RPPN Mata da Serra é um refúgio importante para espécies de mamíferos não voadores da região. Este trabalho contribuiu para o desenvolvimento do Plano de Manejo da área, sobretudo no tocante ao zoneamento da unidade, a ações de reflorestamento e de educação ambiental com os visitantes e vizinhos da unidade.

\section{Inventory of non-flying mammals from the private reserve of natural heritage Mata da Serra (ES)}

The Atlantic Forest stretches from Rio Grande do Norte to Rio Grande do Sul on the coast. It can be divided into two bodies: Coastal Forest and Mountain Forest. Both have a large number of endemism has and have a very high biodiversity and a valuable genetic bank. The establishment of Conservation Units in remaining areas of this biome has become one of the main strategies for maintaining biodiversity. In addition to the creation of these units, it is also necessary to carry out studies that establish their real environmental conservation situation, collaborating to increase the knowledge of the biology of the species belonging to these areas. The objective of this study was to inventory the existing mammals in the PRNH Mata da Serra, in the municipality of Vargem Alta, Espírito Santo, as a way to subsidize the creation of a Management Plan for the area and other improvements for it. In a period of 11 months in the field (from september 2012 to august 2013), 107 animals were registered, divided into 15 species of non-flying mammals and distributed in 05 orders and 11 families. Of the registered species, three are threatened with extinction. The results obtained highlight the importance of conservation units, even those of relatively small area, for connectivity to the maintenance and preservation of biodiversity. They indicate that the PRNH Mata da Serra is an important refuge for non-flying mammal species in the region. This work contributed to the development of the Management Plan for the area, especially regarding the zoning of the unit, reforestation and environmental education actions with visitors and neighbors of the unit.

Keywords: Mammals; Atlantic Forest; Conservation Units.

Topic: Conservação da Biodiversidade

Reviewed anonymously in the process of blind peer.

Luan Gonçalves Bissa (it)

Centro Universitário São Camilo, Brasil

http://lattes.cnpq.br/1550232803551374

http://orcid.org/0000-0001-7262-0805

luan.bio@hotmail.com

Helimar Rabello (iD)

Universidade Federal do Espírito Santo, Brasil

http://lattes.cnpq.br/3789680363736207

http://orcid.org/0000-0002-1699-595X

helimarbio@hotmail.com

Caio Henrique Ungarato Fiorese (ic

Centro Universitário São Camilo, Brasil

http://lattes.cnpq.br/0545804621047516

http://orcid.org/0000-0001-6866-0361

caiofiorese@hotmail.com

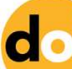

DOI: 10.6008/CBPC2318-2881.2020.002.0001
Received: 10/03/2020

Approved: 12/04/2020

\author{
Gilson Silva Filho (iD) \\ Centro Universitário São Camilo, Brasil \\ http://lattes.cnpq.br/1040363193594196 \\ http://orcid.org/0000-0001-5313-1424 \\ silva.filho.gilson@gmail.com \\ Cíntia Cristina Lima Teixeira \\ Centro Universitário São Camilo \\ http://lattes.cnpq.br/2013098051038491 \\ http://orcid.org/0000-0002-8438-5897 \\ cintiateixeira@saocamilo-es.br \\ Gabrielli Machado Bindeli (iD) \\ Centro Universitário São Camilo, Brasil \\ http://lattes.cnpq.br/3704712429916403 \\ http://orcid.org/0000-0002-3159-4046 \\ gabriellimbindeli@gmail.com
}

\section{Referencing this:}

BISSA, L. G.; RABELLO, H.; FIORESE, C. H. U.; SILVA FILHO, G.; TEIXEIRA, C. C. L.; BINDELI, G. M.. Inventário de mamíferos não voadores da reserva particular do patrimônio natural Mata da Serra (ES). Nature and Conservation, v.13, n.2, p.1-13, 2020. DOI:

http://doi.org/10.6008/CBPC2318-2881.2020.002.0001 


\section{INTRODUÇÃO}

A Floresta Pluvial Atlântica ou Mata Atlântica é um corpo florestal do Brasil oriental, estendendo-se do Rio Grande do Norte até o Rio Grande do Sul na faixa litorânea. A Mata Atlântica pode ser dividida em dois corpos: Floresta Litorânea e Floresta de Montanha. Ambas possuem um grande número de endemismos (SICK, 1997). Ocorre desde o estado do Rio Grande do Norte até o estado do Rio Grande do Sul, chegando até a abranger alguns trechos do Paraguai e da Argentina (SILVA et al., 2005; TABARELLI et al., 2005)

A Mata Atlântica é detentora de uma elevada biodiversidade e de um valioso banco genético, sendo a formação vegetal mais antiga do Brasil, com aproximadamente 70 milhões de anos (LEITÃO-FILHO, 1987). Esse bioma, atualmente, encontra-se muito fragmentado devido a várias ações antrópicas. Com isso, esse território perdeu grande parte de sua cobertura vegetal, restando atualmente algo em torno de $7 \%$ de cobertura florestal nativa. O restante de sua área encontra-se coberta por pastagens, lavouras e áreas urbanas (GOMES et al., 2013). De uma área original distribuída ao longo de 17 estados brasileiros, restam apenas $7,3 \%$ desse total $\left(95.000 \mathrm{Km}^{2}\right.$ ) (SOSMA et al., 1998).

O estabelecimento de Unidades de Conservação (UCs) em áreas remanescentes da Mata Atlântica tem se tornado uma das principais estratégias para a manutenção da biodiversidade nativa. As áreas destinadas à proteção dos diversos ecossistemas necessitam de uso e administração planejados, de modo que sua conservação seja garantida e contemple as finalidades ambientais e científicas (MILANO, 2001). Alguns remanescentes florestais ainda abrigam diversas espécies. No entanto, a riqueza de espécies pode estar fortemente associada com a qualidade e o tamanho do fragmento (REIS et al., 2003). Além da criação dessas unidades, também é necessário que sejam realizados estudos que estabeleçam a real situação de conservação ambiental das mesmas, colaborando para o aumento do conhecimento da biologia das espécies integrantes destas áreas.

Existem 5.478 espécies de mamíferos descritos em todo o mundo (REIS et al., 2006a). Os mamíferos são considerados componentes importantes para os ecossistemas. São, ainda, classificados como bons indicadores de qualidade ambiental (D'ANDREA et al., 1999). O Brasil detém 652 espécies, com cerca de 12\% do total de espécies descritas (REIS et al., 2006b). As espécies de mamíferos que ocorrem no Brasil estão distribuídas em 11 ordens: Marsupiais, Edonatos, Roedores, Lagomorfos, Quirópteros, Cetáceos, Sirênios, Carnívoros, Perissodáctilos, Artiodáctilos e Primatas (CÂMARA et al., 2003).

Algumas características importantes são: a endotermia, visto que possuem mecanismos termorreguladores internos que controlam a temperatura do corpo; o cuidado com a prole, que é mais desenvolvida nesta classe e alcança seu clímax na espécie humana e; o sucesso reprodutivo, que se dá pela capacidade de retenção dos filhotes no trato reprodutivo da fêmea durante um período de tempo mais longo de desenvolvimento em relação às aves que fazem a postura de ovos. Nos mamíferos, esse método significa a produção de menos filhotes, que possuem uma maior chance de sobreviverem (STORER, 1992). Além disso, os mamíferos são os únicos animais que possuem expressão facial, caracterizando-se também por terem heterodontia (dentes morfologicamente diferenciados, ou seja, com formas e funções distintas (ELIAS, 2006) 
e dois conjuntos de dentição em toda a sua vida, que são chamados de difiodontia (POUGH, 2003).

Os mamíferos, de forma geral, apresentam tamanhos variados e necessidades específicas para sua sobrevivência. São especialmente afetados pela fragmentação (BIERREGAARD JUNIOR et al., 1992; DIDHAM, 1997), sendo que isso pode modificar a sua diversidade e abundância. Mudanças em tais parâmetros ocorrem mais rapidamente em fragmentos pequenos do que nos grandes (COSSON et al., 1999). No entanto, muitos aspectos ecológicos e biológicos sobre os mamíferos são, ainda hoje, pouco entendidos. Isso se deve a seus hábitos noturnos e esquivos e pela baixa densidade de suas populações, o que os torna difíceis de observar e estudar (CROOKS, 2002). Apesar de a Mata Atlântica ter uma alta diversidade de espécies com muitas espécies endêmicas, no Estado do Espírito Santo, a fauna de mamíferos é ainda muito pouco conhecida (PASSAMANI et al., 2005).

O principal objetivo deste estudo foi inventariar os mamíferos existentes na RPPN Mata da Serra, em Vargem Alta, Estado do Espírito Santo. Os resultados deste trabalho fizeram parte do plano de manejo da unidade e auxiliaram em tomadas de decisões referentes à mesma, além de complementar os estudos deste grupo de fauna no Espírito Santo e subsidiar diversas outras melhorias na área.

\section{MATERIAIS E MÉTODOS}

O trabalho contou com expedições quinzenais, totalizando, ao final da pesquisa, 24 excursões. Foi desenvolvido durante os meses de Setembro de 2012 a Agosto de 2013, na Reserva Particular do Patrimônio Natural (RPPN) Mata da Serra. A mesma conta com uma área de 14,54 hectares e altitude média em torno de 770 metros. É caracterizada pela predominância da Mata Atlântica Ombrófila Sub-Montana. Situa-se no município de Vargem Alta (Distrito de São Benedito), Estado do Espírito Santo, Brasil (UTM 77177600 /294800 DATUM SAD 69). A Figura 1 apresenta a vista aérea da propriedade desse estudo. A parte hachurada demonstra a região destinada a RPPN Mata da Serra.

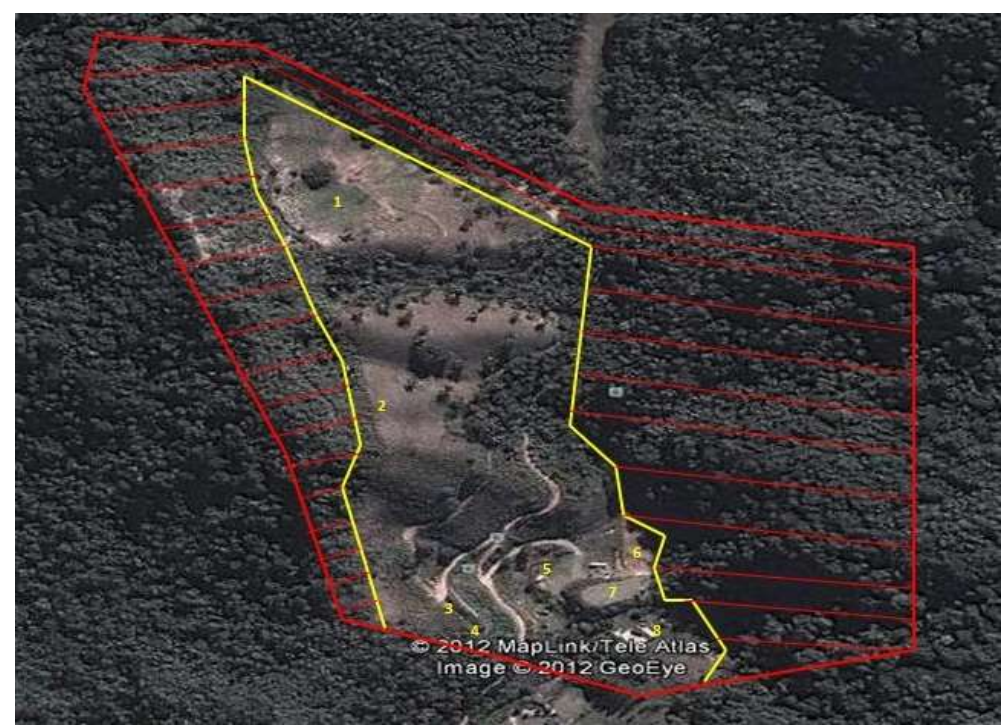

Figura 1: Carta imagem com a área da propriedade.

Os equipamentos utilizados foram: 4 armadilhas fotográficas/filmadoras Bushnell ZT820; Digitais Canon SX10Is e Sony H50; iscas alimentares para atrativo de fauna; câmeras; GPS Garmin Rino 110 para o 
georeferenciamento dos pontos analisados; computador de mão Acer Aspire 5538 para registro de imagens capturadas; fitas métricas; lápis e caderneta de campo. Na identificação das espécies, foi utilizado o material bibliográfico de Paz et al. (2003) e Reis (2011). A identificação dos vestígios foi feita com base no Manual de Rastros (MORO-RIOS, 2008) e através do material bibliográfico de Becker et al. (1991).

A metodologia aplicada foi baseada em busca ativa por vestígios, uso de armadilhas fotográficas e através da utilização de 24 armadilhas de gaiola modelo Tomahawk $45 \times 17,5 \times 15 \mathrm{~cm}$. Para avaliar as comunidades, diversos métodos foram empregados, entre os quais as armadilhas fotográficas, o acervo fotográfico pessoal e registros de sinais (Figura 2), como pegadas e fezes (ZAPATA-RIOS, 2006). Nas buscas ativas, foram percorridas trilhas e estradas preexistentes em períodos matutinos ( $5.00 \mathrm{~h}$ às $8.00 \mathrm{~h}$ ) e vespertinos (16.00h às $18.00 \mathrm{~h}$ ). Foram feitos registros de vestígios, como fezes, pelos, tocas e pegadas com confecção de moldes de gesso das mesmas.
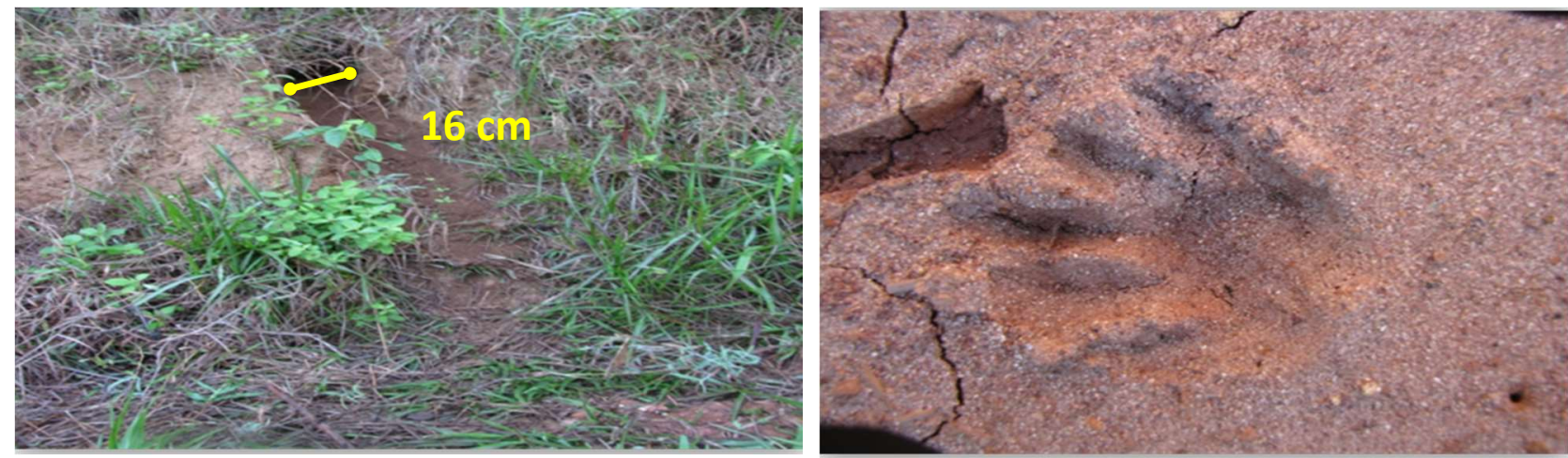

Figura 2: Vestígios de Mamíferos: Trilha Mamífero de Pequeno Porte - Pegada de Mão-pelada (Procyon cancrivorus).

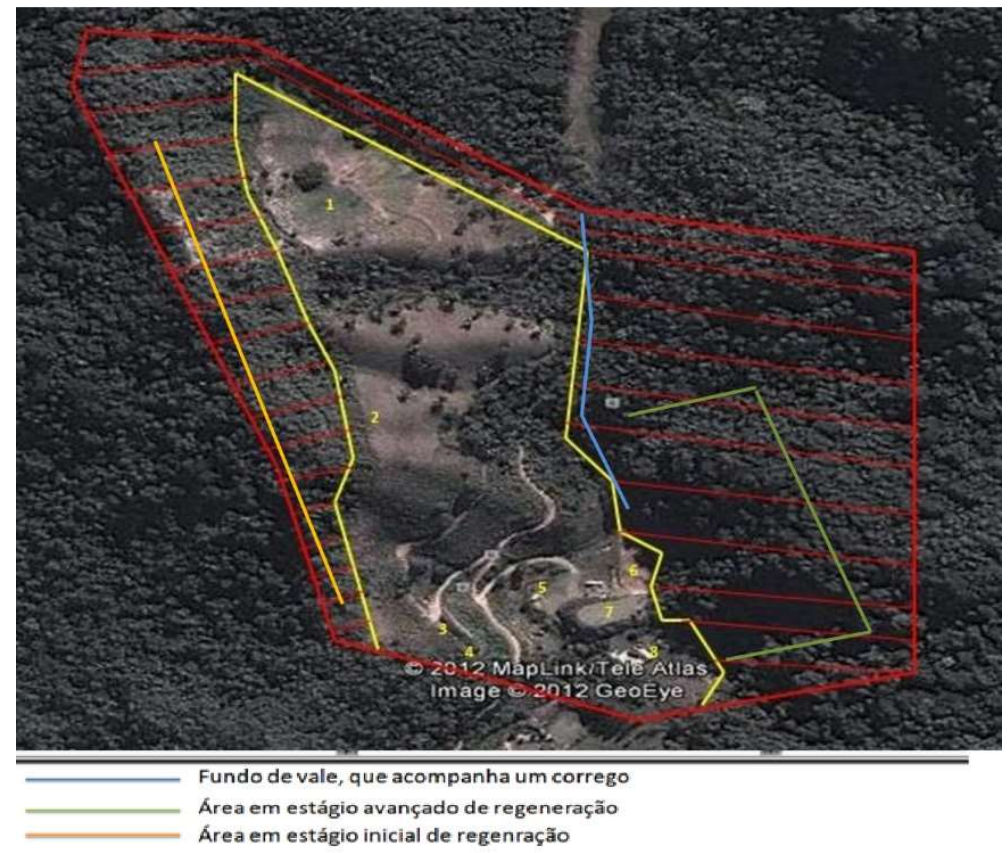

Figura 3: Carta imagem com a área da propriedade com marcação dos transectos.

Para a colocação das gaiolas e armadilhas fotográficas foram efetuados três transectos, sendo dois em vertentes de morros. 0 primeiro foi instalado em uma área em estágio avançado de regeneração. Já o segundo em uma área em estágio inicial de regeneração e o terceiro, em um fundo de vale que acompanha um córrego existente, com vegetação também em estágio avançado. Foram empregadas 8 gaiolas em cada 
um, a uma distância de 30 metros uma da outra. A Figura 3 demonstra os locais onde os transectos foram definidos.

Para as armadilhas iscadas, foram utilizadas frutas, verduras, pasta de amendoim, carne bovina (provenientes do descarte da limpeza de carnes) e sardinha. $O$ tipo de isca foi trocado a cada expedição, ou seja, em uma era utilizado determinado tipo de fruta e carne, em outra expedição era utilizada outro tipo de fruta e carne. Após a coleta em campo, os dados foram levados para o laboratório de Zoologia da instituição de ensino 'Centro Universitário São Camilo', no município de Cachoeiro de Itapemirim, Estado do Espírito Santo, onde foram separados, triados e as espécies, identificadas.

\section{RESULTADOS E DISCUSSÃO}

Em um período de 11 meses de campo (de setembro de 2012 a agosto de 2013), foram registrados, neste trabalho, 107 animais divididos em 15 espécies de mamíferos não voadores, distribuídas em 05 ordens e 11 famílias. As ordens com maior número de espécies coletadas foram: Carnívora ( $n=7$ ) com 46,66\% e sendo a mais representativa; Primates $(n=3)$, com 20\%; Didelphimorphia $(n=2)$, com $13,33 \%$; Rodentia $(n=2)$, com 13,33\% e; Cingulata ( $n=1$ ), com 6,66\%. A Figura 4 faz referência a porcentagem de espécies por ordem.

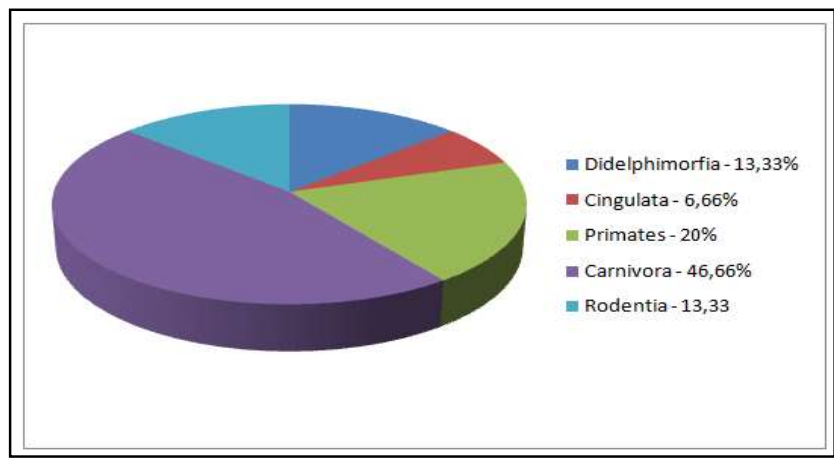

Figura 4: Quantidade de espécies por ordem.

Quanto às famílias Procyonidae, Mustelidae, Didelphidae e Felidae, foram registradas 02 espécies e, as demais, somente 1 espécie em cada família. A ordem carnívora, com 07 espécies, foi a mais representativa, conforme se observa na Tabela 1.

Tabela 1: Relação de ordens, família, espécie e nome comum de setembro de 2012 a agosto de 2013 na RPPN Mata da Serra.

\begin{tabular}{|l|l|l|l|}
\hline Ordem & Família & Classificação Sistemática & Nome Comum \\
\hline \multirow{3}{*}{ Didelphimorphia } & Didelphidae & Didelphis aurita (WIED-NEUWIED, 1826) & Gambá \\
\cline { 2 - 4 } & & Philander frenatus (OLFERS, 1818) & Cuíca \\
\hline Cingulata & Dasypodidae & Dasypus novemcinctus (LINNAEUS, 1758) & Tatu Galinha \\
\hline Primates & Cebidae & Callithrix flaviceps (THOMAS, 1903) & Sagui da Serra \\
\cline { 2 - 4 } & Phiteciidae & Callicebus sp. & Guigó \\
\cline { 2 - 4 } & Atelidae & Alouatta guariba (HUMBOLDT, 1812) & Bugio/Barbado \\
\hline \multirow{5}{*}{ Carnívora } & Felidae & Puma concolor (GOLDMAN, 1946) & Onça-parda / Suçuarana \\
\cline { 2 - 4 } & & Leopardus pardalis (LINNAEUS, 1758) & Jaguatirica \\
\cline { 2 - 4 } & Canidae & Cerdocyon thous (LINNAEUS, 1766) & Cachorro do Mato \\
\cline { 2 - 4 } & Mustelidae & Eira barbara (LINNAEUS, 1758) & Furão \\
\cline { 2 - 4 } & & Galictis sp. & Quati \\
\cline { 2 - 4 } & Procyonidae & Nasua nasua (LINNAEUS, 1766)) & Caxinguelê \\
\cline { 2 - 3 } & & Procyon cancrivorus (G. [Baron] CUVIER, 1798) \\
\hline
\end{tabular}


Deve-se destacar que, das 03 espécies de primatas registradas, todas são endêmicas do Bioma Mata Atlântica e estão inseridas no Plano de Ação Nacional para a Conservação dos Mamíferos da Mata Atlântica Central, aprovado por meio da Portaria № 134 de 23/12/2010 do ICMBio (Instituto Chico Mendes de Conservação da Biodiversidade) (BRASIL, 2016).
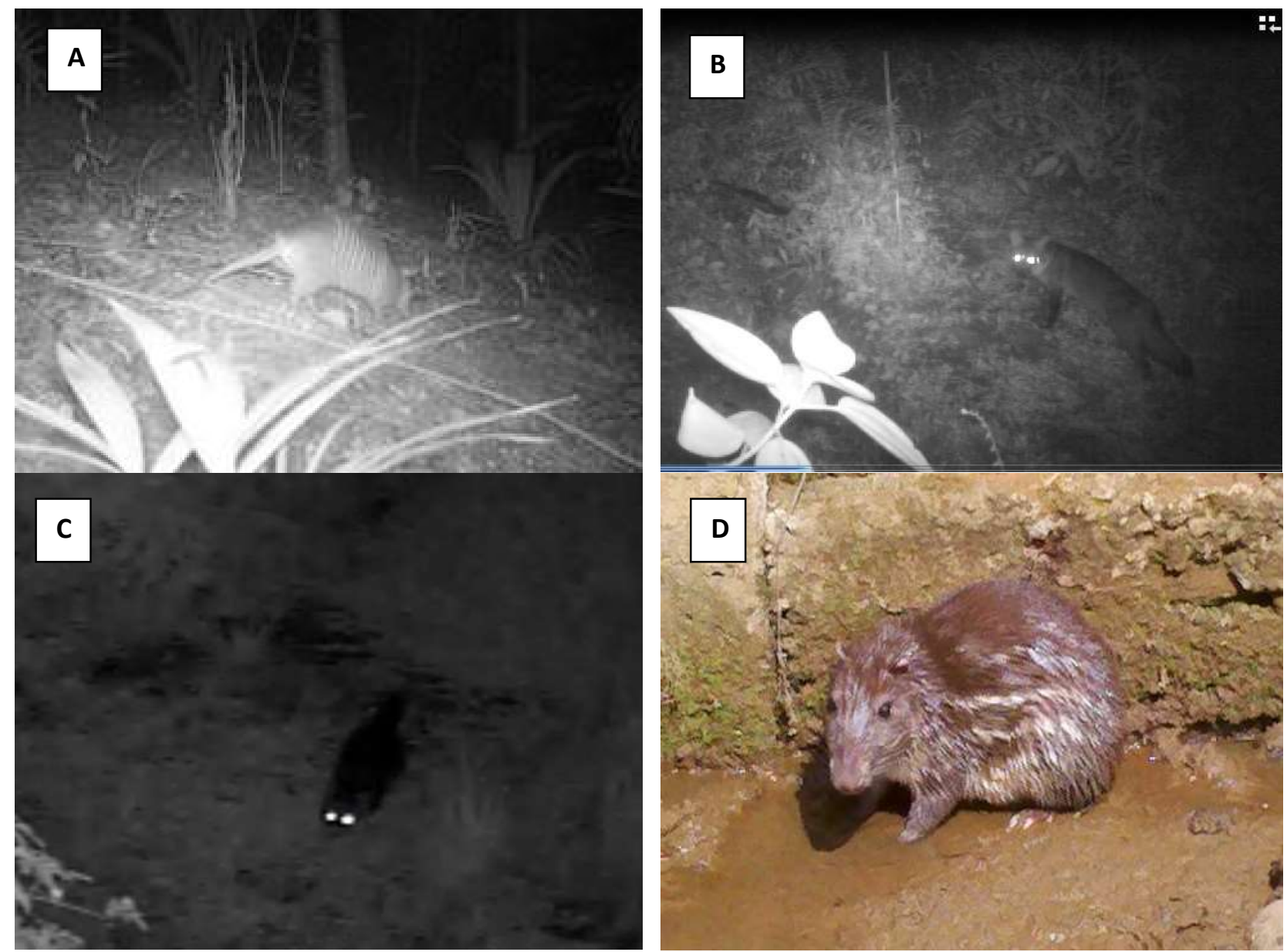

Figura 5: A: Tatu Galinha (Dasypus novemcinctus); B: Cachorro do mato (Cerdocius thous); C: Mão Pelada (Procyon cancrivorus); D: Paca (Cuniculus paca).

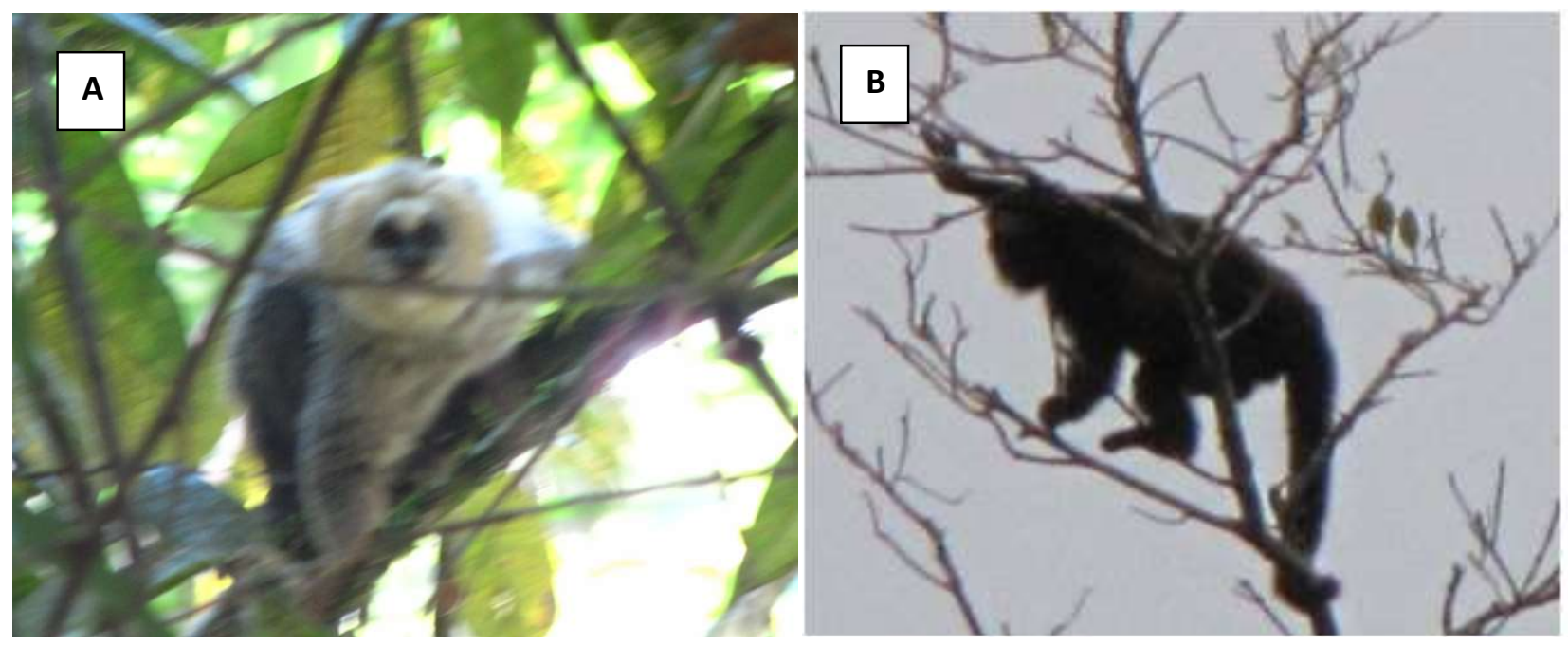

Figura 6: A: Sagui da Serra (Callithrix flaviceps); B: Bugio/Barbado (Alouatta guariba).

Descrição das espécies registradas e ameaçadas de extinção - Callithrix flaviceps: Encontra-se com status 'em perigo' no estado do Espírito Santo e 'ameaçada', no Livro vermelho de Espécies Ameaçadas do 
Ministério do Meio Ambiente (BRASIL, 2008). Espécie inserida na lista (Mundial) da International Union for Conservation of Nature (IUCN, 2007) com status 'em perigo'. Esta espécie foi utilizada como espécie bandeira da RPPN Mata da Serra. Alouatta guariba: Encontra-se criticamente em Perigo (CR) (BRASIL, 2008). Callicebus sp.: Encontra-se como vulnerável (VU) (BRASIL, 2008). A Figura 5 demonstra algumas imagens de espécies registradas e retiradas das armadilhas fotográficas e a Figura 6 mostra duas das três espécies ameaçadas de extinção e que foram registradas através de armadilhas fotográficas.

As Tabelas 2 e 3 mostram as descrições das espécies encontradas os valores estimados referentes a: número de indivíduos, número de encontros da espécie, frequência de ocorrência (FO), classe de frequência, abundância relativa (AR), abundância e classe de abundância (CTA). Calculou-se a FO dividindo o número de registros de uma determinada espécie durante todas as campanhas pela quantidade de campanhas e, em seguida, multiplicando-se por 100. O cálculo para se chagar a AR ocorreu através da divisão do número de indivíduos de uma determinada espécie pelo número total de todos os indivíduos encontrados ao final de todas as campanhas e, posteriormente, pela divisão deste resultado por 100.

Tabela 2: Relação de espécie e número de indivíduos, número de encontros de espécies, frequência de ocorrência e classe de frequência registrada nos meses de setembro de 2012 a agosto de 2013 na RPPN Mata da Serra, Vargem Alta, ES. Classe de Frequência; PF: Pouco Frequente / F: Frequente / MF: Muito Frequente.

\begin{tabular}{|c|c|c|c|c|}
\hline Espécies encontradas & № de indivíduos & № de encontros da sp. & Freq. de ocorrência (FO) & Classe de Frequência \\
\hline Didelphis aurita & 18 & 16 & 133,33 & $\mathrm{MF}$ \\
\hline Philander frenatus & 11 & 11 & 91,67 & MF \\
\hline Dasypus novemcinctus & 13 & 12 & 100,00 & MF \\
\hline Callithrix flaviceps & 5 & 3 & 25,00 & F. \\
\hline Callicebus personatus & 14 & 4 & 33,33 & F. \\
\hline Alouatta clamitans & 10 & 8 & 66,67 & MF \\
\hline Cebus nigritus & 3 & 3 & 25,00 & $\mathrm{~F}$. \\
\hline Puma concolor & 1 & 1 & 8,33 & PF \\
\hline Leopardus pardalis & 1 & 1 & 8,33 & $\mathrm{PF}$ \\
\hline Leopardus tigrinus & 1 & 1 & 8,33 & PF \\
\hline Cerdocyon thous & 7 & 7 & 58,33 & MF \\
\hline Eira barbara & 3 & 3 & 25,00 & F. \\
\hline Galictis sp. & 3 & 3 & 25,00 & F. \\
\hline Nasua & 4 & 4 & 33,33 & F. \\
\hline Procyon cancrivorus & 2 & 2 & 16,67 & $\mathrm{PF}$ \\
\hline Guerlinguetus ingrami & 8 & 8 & 66,67 & MF \\
\hline Cuniculus paca & 3 & 3 & 25,00 & F. \\
\hline
\end{tabular}

Tabela 3: Relação de espécie e abundância, abundância relativa e classe de abundância registrada nos meses de setembro de 2012 a agosto de 2013 na RPPN Mata da Serra, Vargem Alta, ES. Abundância; PA: Pouco Abundante/ Abundância A: Abundante/ MA: Muito Abundante/ Classe de Abundância; A: Abundante/ C: Comum/ R: Raro.

\begin{tabular}{|l|l|l|l|}
\hline Espécies encontradas & Abundância Relativa (AR) & Abundância & Classe de Abundância (CTA) \\
\hline Didelphis aurita & 16,82 & MA & A \\
\hline Philander frenatus & 10,28 & MA & A \\
\hline Dasypus novemcinctus & 12,15 & MA & A \\
\hline Callithrix flaviceps & 4,67 & A. & MA \\
\hline Callicebus personatus & 13,08 & MA & A \\
\hline Alouatta clamitans & 9,35 & A. & C \\
\hline Cebus nigritus & 2,80 & PA & R \\
\hline Puma concolor & 0,93 & PA & R \\
\hline Leopardus pardalis & 0,93 & PA & A \\
\hline Leopardus tigrinus & 0,93 & MA & A \\
\hline Cerdocyon thous & 6,54 & A. & C \\
\hline Eira barbara & 2,80 & A. & C \\
\hline Galictis sp. & 2,80 & A. & C \\
\hline Nasua nasua & 3,74 & & \\
\hline
\end{tabular}




\begin{tabular}{|l|l|l|l|}
\hline Procyon cancrivorus & 1,87 & PA & R \\
\hline Guerlinguetus ingrami & 7,48 & MA & A \\
\hline Cuniculus paca & 2,80 & A. & C \\
\hline
\end{tabular}

Ao analisar a tabela acima se deve dar uma maior atenção para o mico-da-serra (Callithrix flaviceps), que é um animal raro e difícil de se encontrar. Porém, na RPPN Mata da Serra, ele é um animal comum e encontrado com certa frequência. Em relação à diversidade da área, foi calculado o índice de diversidade Shannon-Wiener $\left(H^{\prime}\right)$. Esse índice, segundo Wihlm (1972), expressa a importância relativa de cada espécie e não apenas a proporção entre espécies e indivíduos. Para calcular esses índices, foram utilizados os dados da tabela acima e os programas Past e Estimate S. A Figura 12 demonstra a curva de diversidade de espécies da RPPN Mata da Serra.

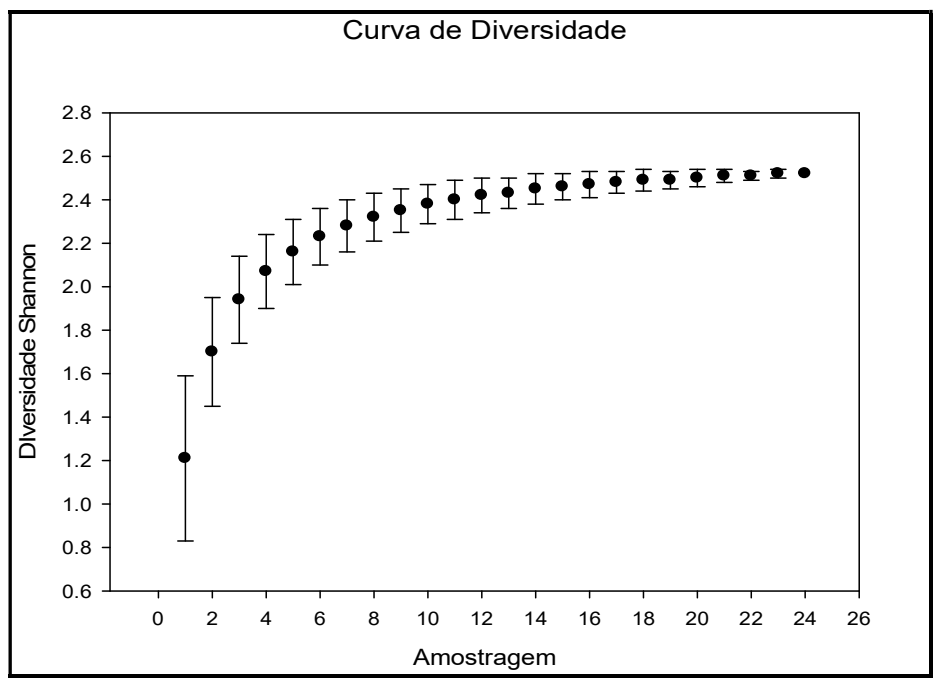

Figura 7: Curva de diversidade de espécies.

Como observado, a curva chegou próximo a estabilidade, porém, ao final do período estudado, houve uma elevação da curva para a área, significando que há tendência de amostragem de novas espécies para o local. O índice de diversidade de Shanon foi de 2,52 tendendo a estabilização, o que vem de encontro ao resultado do indicador Jackniffe, demostrando um bom esforço amostral. O índice de Magalef $(3,424)$ também confirma uma riqueza de espécies nos resultados deste trabalho, com uma equitabilidade alta e uma diversidade alta, conforme demonstra a Tabela 4.

Quanto à equitabilidade, que foi de 0,8902, demonstrou que o número de indivíduos dentro de cada espécie foi similar, o que foi confirmado através da estimativa do Índice de Berger-Parker, que foi de 0,1682. Assim, foi confirmada a ausência de uma espécie dominante nos resultados. A Figura 7 apresenta a curva de rarefação para riqueza de espécies da RPPN Mata da Serra.

Tabela 4: Dados estatísticos da RPPN Mata da Serra, Vargem Alta, ES.

\begin{tabular}{|l|l|}
\hline $\mathbf{0}$ & $\mathbf{A}$ \\
\hline Taxa_S & 15 \\
\hline Individuals & 107 \\
\hline Shannon_H & 2,522 \\
\hline Margalef & 3,424 \\
\hline Equitability_J & 0,8902 \\
\hline Berger-Parker & 0,1682 \\
\hline
\end{tabular}




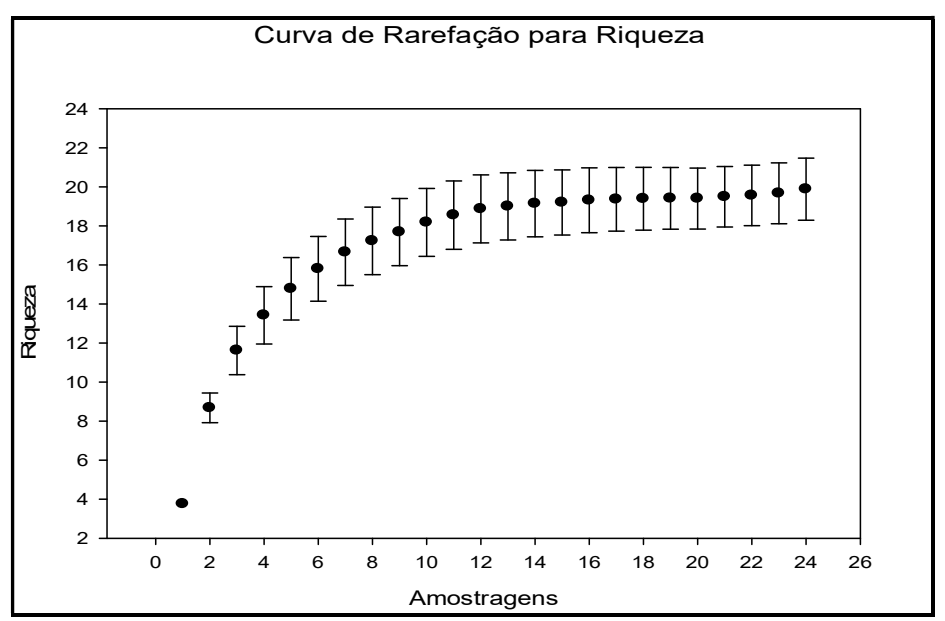

Figura 8: Curva de rarefação para riqueza de espécies.

Segundo o indicador Jackniffe-1, o valor foi 19,87 e o erro, igual a 1,58. Dessa forma, seria possível coletar, aproximadamente, pelo menos mais uma espécie e no máximo 4, caso o esforço amostral fosse de 100 idas a campo. Assim, o resultado foi satisfatório para o uso no plano de manejo da unidade. Do total de espécies, 10 foram registradas através de registro visual, sendo que, destas, 6 tiveram apenas esse tipo de registro e 4 apenas através de registro de armadilha fotográfica. Das 15 espécies registradas, 5 foram confirmadas por dois ou mais métodos empregados na metodologia (Registro Visual, Registro Fotográfico, Armadilha Fotográfica e Pegadas) conforme demonstram a Tabela 5 e a Figura 9.

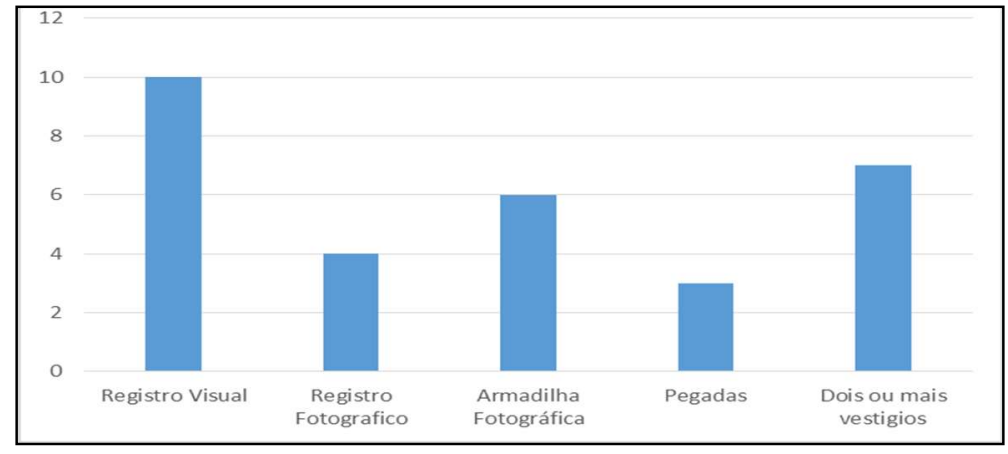

Figura 9: Tipos de registro.

Tabela 5: Relação de espécie e tipos de registros de 2012 a agosto de 2013 na RPPN Mata da Serra, Vargem Alta, ES; 1 - Registro Visual / 2 - Registro Fotográfico / 3 -Armadilha Fotográfica / 4 - Pegadas.

\begin{tabular}{|l|l|l|}
\hline Classificação Sistemática & Nome Comum & Tipos de Registro \\
\hline Didelphis aurita (WIED-NEUWIED, 1826) & Gambá & 1 \\
\hline Philander frenatus (OLFERS, 1818) & Cuíca & 1 \\
\hline Dasypus novemcinctus (LINNAEUS, 1758) & Tatu Galinha & $1-2-3$ \\
\hline Callithrix flaviceps (THOMAS, 1903) & Sagui da Serra & $1-2$ \\
\hline Callicebus sp. & Guigó & 1 \\
\hline Alouatta guariba (HUMBOLDT, 1812) & Bugio/Barbado & $1-2$ \\
\hline Puma concolor (GOLDMAN, 1946) & Onça-parda / Suçuarana & $3-4$ \\
\hline Leopardus pardalis (LINNAEUS, 1758) & Jaguatirica & $3-4$ \\
\hline Cerdocyon thous (LINNAEUS, 1766) & Cachorro do Mato & 3 \\
\hline Eira barbara (LINNAEUS, 1758) & Irara & 1 \\
\hline Galictis sp. & Furão & 1 \\
\hline Nasua nasua (LINNAEUS, 1766)) & Quati & 1 \\
\hline $\begin{array}{l}\text { Procyon cancrivorus (G. [Baron] CUVIER, } \\
\text { 1798) }\end{array}$ & Mão Pelada & $3-4$ \\
\hline Guerlinguetus ingrami (THOMAS, 1901) & Caxinguelê & 3 \\
\hline Cuniculus paca (LINNAEUS, 1758) & Paca & 1 \\
\hline
\end{tabular}


Em relação ao uso de habitat, houve dominância de mamíferos terrestres, com um total de 7 espécies, seguido de arborícola, com 4 espécies, e cursorial, com 4. A Tabela 6 e a Figura 10 demonstram os registros quanto ao uso de habitat pelas espécies registradas.

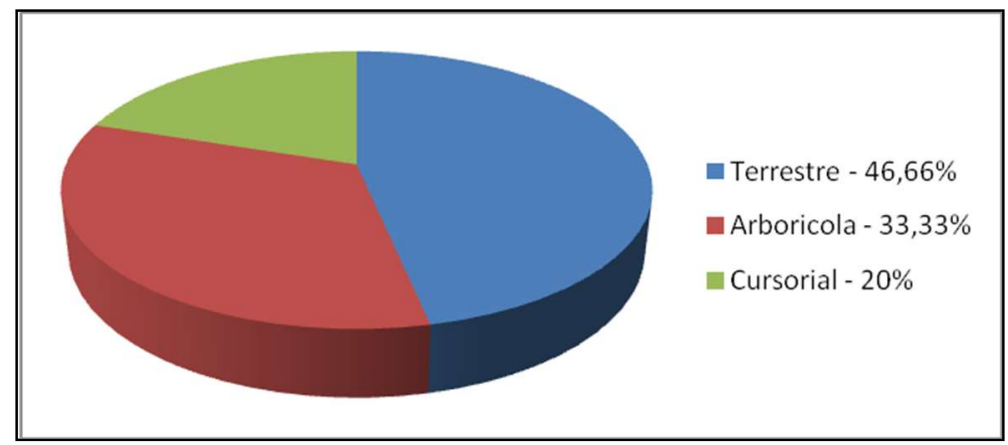

Figura 10: Uso de habitat pelos animais.

Tabela 6: Relação de espécies e tipos de habitat.

\begin{tabular}{|l|l|l|}
\hline \multicolumn{1}{|c|}{ Classificação Sistemática } & Nome Comum & Tipos de Habitat \\
\hline Didelphis aurita (WIED-NEUWIED, 1826) & Gambá & Cursorial \\
\hline Philander frenatus (OLFERS, 1818) & Cuíca & Cursorial \\
\hline Dasypus novemcinctus (LINNAEUS, 1758) & Tatu Galinha & Terrestre \\
\hline Callithrix flaviceps (THOMAS, 1903) & Sagui da Serra & Arborícola \\
\hline Callicebus sp. & Guigó & Arborícola \\
\hline Alouatta guariba (HUMBOLDT, 1812) & Bugio/Barbado & Arborícola \\
\hline Puma concolor (GOLDMAN, 1946) & Onça-parda / Suçuarana & Terrestre \\
\hline Leopardus pardalis (LINNAEUS, 1758) & Terrestre \\
\hline Cerdocyon thous (LINNAEUS, 1766) & Jaguatirica & Terrestre \\
\hline Eira barbara (LINNAEUS, 1758) & Cachorro do Mato & Cursorial \\
\hline Galictis sp. & Irara & Terrestre \\
\hline Nasua nasua (LINNAEUS, 1766)) & Furão & Cursorial \\
\hline Procyon cancrivorus (G. [Baron] CUVIER, 1798) & Quati & Terrestre \\
\hline Guerlinguetus ingrami (THOMAS, 1901) & Mão Pelada & Arborícola \\
\hline Cuniculus paca (LINNAEUS, 1758) & Caxinguelê & Terrestre \\
\hline
\end{tabular}

Tendo em vista que a RPPN Mata da Serra é um fragmento pequeno que, durante muito tempo, sofreu fortes ações antrópicas e hoje se encontra em um estágio secundário de regeneração, o presente estudo obteve um resultado satisfatório, tendo em vista que a quantidade de espécies encontradas na área pode ser comparada a áreas maiores, baseando-se em trabalhos com o mesmo objetivo. Um exemplo que pode ser citado é o trabalho realizado na RPPN da Universidade de Santa Cruz do Sul (UNISC) (ABREU JÚNIOR et al., 2009), que conta com uma área de 221,39 hectares e apresenta um resultado de 16 espécies encontradas. Já A RPPN Mata da Serra apresenta uma área de apenas 7,01\% da área da RPPN da UNISC e contou com 15 espécies em seu resultado final. Outra comparação a ser feita foi com o trabalho no Parque Estadual Mata São Francisco (PEMSF), localizada entre os municípios de Cornélio Procópio e Santa Mariana, estado do Paraná (MEIGA et al., 2008), onde o local de pesquisa apresentava uma área de 832,57 hectares e apresentou em seu resultado final 12 espécies.

Das 250 espécies que ocorrem na Mata Atlântica, 135 ocorrem no Espírito Santo, incluindo a ordem Chiroptera (FONSECA et al., 1996). Ao analisar esses dados e comparar com outros trabalhos realizados, como o estudo feito nas áreas de reserva legal da fazenda Brunoro Agro avícola, situada no município de 
Venda Nova do Imigrante - Espírito Santo (RABELLO et al., 2009), com uma área total de 12,85 hectares e sua utilização como corredor ecológico para passagem de outros animais, este apresentou, em seu resultado final, 20 espécies. Apesar da RPPN Mata da Serra apresentar um tamanho maior, a área da fazenda Brunoro tem a função de servir como corredor ecológico o que pode justificar a presença de um número maior de espécies.

Os levantamentos faunísticos em fragmentos florestais são de suma importância e essenciais para avaliar o efeito de fragmentação sobre a diversidade de mamíferos e a perturbação nos remanescentes florestais (D'ANDREA et al., 1999). A presença de 15 espécies de mamíferos não voadores encontrados na RPPN estudada demonstra que áreas protegidas são importantes para manter certas espécies de fauna local. Entretanto, segundo Voss et al. (1996), levantamentos feitos em longo prazo (mais de dois anos) considerando o uso de outras técnicas de amostragens (armadilha de queda, por exemplo) são necessários, a fim de que amostragens se tornem mais completas. Para o desenvolvimento de práticas conservacionistas, é de suma importância o conhecimento das espécies ali presentes e da sua distribuição espacial (KASPER et al., 2007).

\section{CONCLUSÕES}

Verificou-se que a RPPN Mata da Serra apresentou um registro de 15 espécies de mamíferos não voadores, sendo que desses, três estão ameaçados de extinção. Assim, mostrou que esses números são satisfatórios, pois a área territorial é relativamente pequena quando comparada com outros estudos realizados em outros locais do estado e, até mesmo, do país, e com o mesmo objetivo. Isso indicou que a área é um refúgio importante para espécies de mamíferos não voadores da região.

Os resultados obtidos, principalmente em relação à presença de espécies raras e ameaçadas na área da RPPN Mata da Serra, ressaltam a importância das unidades de conservação, mesmo aquelas de área relativamente pequena, para conectividade a manutenção e preservação da biodiversidade. Estes dados contribuíram para o desenvolvimento do Plano de Manejo da unidade estudada, sobretudo no tocante ao seu zoneamento, a ações de reflorestamento e de educação ambiental com os visitantes e vizinhos da unidade, pois para isso foi necessário o conhecimento das espécies ocorrentes na região. Assim, para o desenvolvimento de ações conservacionistas na RPPN Mata da Serra (ES), é necessário conhecer as espécies e de suas distribuições no espaço.

\section{REFERÊNCIAS}

ABREU JUNIOR, E. F.; KÖHLER, A.. Mastofauna de médio e grande porte na RPPN da UNISC, RS, Brasil. Biota Neotropica, Campinas, v.9, n.4, 2009.

BECKER, M.; DALPONTE, J. C.. Rastros de Mamíferos Silvestres brasileiros: um guia de campo. 2 ed. Brasília: Universidade de Brasília, 1991.

BIERREGAARD JUNIOR, R. O.; LOVEJOY, T.; KAPOS, V.; SANTOS, A. A.; HUTCHINGS, R. W.. The Biological dynamics of tropical rainforest fraguements. Biosciences, v.42, n.11, p.859-866, 1992

BRASIL. Ministério do Meio Ambiente. Livro vermelho da fauna brasileira ameaçada de extinção. Brasília: Ministério do Meio Ambiente; Belo Horizonte: Fundação Biodiversitas, 2008.

BRASIL. Ministério do Meio Ambiente. Plano de ação nacional para conservação dos mamíferos da Mata Atlântica Central. Brasília: ICMBIO, 2016. 
CÂMARA, T.; MURTA, R.. Mamíferos da Serra de Cipó. Belo Horizonte: Bicho do Mato, 2003.

COSSON, J.; DREANNO, C.; BILLARD, R.; SUQUET, M.; CIBERT, C.. Regulation of axonemal wave parameters of fish spermatozoa by ionic factors. In: GAGNON, C.. The male gamete: From basic science to clinical applications. Paris: Cache River Pres, 1999.

CROOKS, K.. Relative sensitivities of mammalian carnivores to habitat fragmentation. Conservation Biology, v.16, p.488502, 2002.

D'ANDREA, P. S.; GENTILE, R.; CERQUEIRA, R.; GRELLE, C. E.; HORTA, C.; REY, L.. Ecology of small mammals in a Brazilian rural area. Revista Brasileira de Zoologia, v.3, n.16, p.611620, 1999.

DIDHAM, R. K.. The influence of edge effects and forest fragmentation on leaf litter invertebrates in Central Amazonia. In: LAURANCE, W. F.; BIERREGAARD JUNIOR, R. O.. Tropical forest remnants: ecology, management, and conservation of fragmented communities. Chicago: University of Chicago Press, 1997.

ELIAS, F. A.. Dentes de amniotas da Lage do Coringa (formação Alcântara, albo-cenomaniano da Bacia de São Luís-Grajaú): identificação, descrição, aspectos paleobiológicos, biocronológicos, paleogeográficos e paleobiogeográficos. Dissertação (Mestrado em Geociências) - Universidade Estadual Paulista, Rio Claro, 2006.

FONSECA, G. A. B.; HERMANN, G.; LEITE, Y. L. R.; MITTERMEIER, R. A.; RYLANDS, A. B.; PATTON, J. L.. Lista anotada dos mamíferos do Brasil. Belo Horizonte: Ocasional Papers, 1996.

GOMES, O. V. O.; MARQUES, E. D.; SOUZA, M. D. C.; SILVA FILHO, E. V.. Influência antrópica nas águas superficiais da cidade de Três Rios, RJ. Geochimica Brasiliensis, v.27, n.1, p.77-86, 2013. DOI: http://dx.doi.org/10.5327/Z0102$\underline{9800201300010007}$

IUCN. International Union for Conservation of Nature. Red List of Threatened Species. Gland: IUCN, 2007.

KASPER, C. B.; FELDENS, M. J.; MAZIM, F. D.; SCHNEIDER, A.; CADEMARTORI, C. V.; GRILLO, H. C. Z.. Mamíferos do Vale do Taquari, Região Central do Rio Grande do Sul. Biociências, Porto Alegre, v.15, n.1, p.53-62, 2007.

LEITÃO FILHO, H. F.. Espécies do gênero brachiaria Griseb nativas e exóticas cultivadas no Estado de São Paulo. Campinas: CATI, 1977.

MEIGA, A. Y. Y.; PIMENTA, M. C. G.; ORSI, M. L.. Levantamento da mastofauna do Parque Estadual Mata São Francisco, estado do Paraná. Londrina: Universidade Filadélfia de Londrina, 2008.

MITTERMEIER, R. A.; COIMBRA FILHO, A. F.; CONSTABLE, I. D.; RYLANDS, A. B.; VALLE, C.. Conservation of primates in the Atlantic Forests of Brazil. International Zoological Yearbook, v.22, n.1, p.2-17, 1982.

MILANO, M. S.. Conceitos e princípios gerais de ecologia e conservação. Curitiba: FBPN, 2001.
MORO-RIOS, R. F.; SILVA-PEREIRA, J. E.; SILVA, P. W.; MOURA-BRITTO, M.; PATROCÍNIO, D. N. M.. Manual de rastros da fauna paranaense. Curitiba: Instituto Ambiental do Paraná, 2008.

PASSAMANI, M.; DALMASCHIO, J.; LOPES, S. A.. Mamíferos não-voadores em áreas com predomínio de Mata Atlântica da Samarco Mineração S.A., município de Anchieta, Espírito Santo. Biotemas, v.18, n.1, p.135-149, 2005. DOI: https://doi.org/10.5007/\%25x

PAZ, P. R.; VENTURINI, A.. Guia ilustrado de mamíferos da Reserva Natural da Vale do Rio Doce. Vila Velha: Originalis Natura, 2003.

POUGH, H.; JANIS, C. M.; HEISER, J. B.. A vida dos vertebrados. 3 ed. São Paulo: Atheneu, 2003.

RABELLO, H.; LACCHINE, P. S.; PINHEIRO, P. C.; MOULIN, E.; VERISSIMO, A.. Levantamento da mastofauna nas áreas de reserva legal da Fazenda Brunoro Agro - Avicola, situada em Venda Nova do Imigrante - Espírito Santo, Brasil. In: CONGRESSO DE ECOLOGIA DO BRASIL, 9. Anais. São Lourenço: Sociedade de Ecologia do Brasil, 2009.

REIS, N. R.; BARBIERI, M. L. S.; LIMA, I. P.; PERACCHI, A. L.. O que é melhor para manter a riqueza dos morcegos (Mammalia, Chiroptera): um fragmento florestal grande ou vários fragmentos de pequeno tamanho?. Revista Brasileira de Zoologia, Curitiba, v.20, n.2, p.225-230, 2003.

REIS, N. R.; OLIVEIRA FILHO, H.; SILVEIRA, G.. Ordem Lagomorpha. Londrina: Mamíferos do Brasil, 2006a.

REIS, N. R.; PERACCHI, A. L.; PEDRO, W. A.; LIMA, I. P.. Mamíferos do Brasil. Londrina: Universidade Estadual de Londrina, 2006b.

REIS, N. R.; PERACCHI, A. L.; PEDRO, W. A.; LIMA, I. P.. Mamíferos do Brasil. 2 ed. Londrina, 2011.

SICK, H.. Ornitologia brasileira. Rio de Janeiro: Nova Fronteira, 1997.

SILVA, J. M. C.; CASTELETI, C. H. M.. Estado da biodiversidade da Mata Atlântica brasileira. In: GALINDO-LEAL, C.; CÂMARA, I. G.. Mata Atlântica: biodiversidade, ameaças e perspectivas. São Paulo: SOSMA; Belo Horizonte: Conservação Internacional, 2005. p.43-59.

SOSMA; INPE; ISA. Fundação SOS Mata Atlântica; Instituto Nacional de Pesquisas Espaciais; Instituto Socioambiental. Atlas da evolução dos remanescentes florestais da Mata Atlântica e ecossistemas associados no período de 19901995. São Paulo: SOSMA, 1998.

STORER, R. W.. The mamals of North America. Philadelphia: Online Publication, 1992.

TABARELLI, M.; PINTO, L. P.; SILVA, J. M. C.; HIROTA, M. M.; BEDÊ, L. C.. Desafios e oportunidades para a conservação da biodiversidade na Mata Atlântica brasileira.

Megadiversidade, v.1, n.1, p.133-138, 2005.

VOSS, R. S.; EMMONS, L. H.. Mammalian diversity in neotropical lowland rainforests: a preliminary assessment. 
New York:

Bulletin of the American Museum of Natural History, 1996.

WILHM, J.. Graphic and mathematical analyses of biotic communities in polluted streams. Annual Review of Entomology, v.17, p.223-252, 1972.
ZAPATA-RIOS, G.; ARAGUILLIN, E.; JORGENSON, J. P.. Caracterización de lacomunidad de mamíferos no voladores em las estribaciones orientales de la Cordillera del Kutukú, Amazônia Equatoriana. Mastozoología Neotropical, v.13, n.2, p.227-238, 2006.

A CBPC - Companhia Brasileira de Produção Científica (CNPJ: 11.221.422/0001-03) detém os direitos materiais desta publicação. Os direitos referem-se à publicação do trabalho em qualquer parte do mundo, incluindo os direitos às renovações, expansões e disseminações da contribuição, bem como outros direitos subsidiários. Todos os trabalhos publicados eletronicamente poderão posteriormente ser publicados em coletâneas impressas sob coordenação da Sustenere Publishing, da Companhia Brasileira de Produção Científica e seus parceiros autorizados. Os (as) autores (as) preservam os direitos autorais, mas não têm permissão para a publicação da contribuição em outro meio, impresso ou digital, em português ou em tradução. 